\title{
Kinks and nanofriction: Structural phases in few-atom chains
}

\author{
Dorian A. Gangloff $\odot,{ }^{1, *}$ Alexei Bylinskii, ${ }^{2}$ and Vladan Vuletic ${ }^{3, \dagger}$ \\ ${ }^{1}$ Cavendish Laboratory, University of Cambridge, JJ Thomson Avenue, Cambridge CB3 OHE, United Kingdom \\ ${ }^{2}$ Department of Chemistry and Chemical Biology and Department of Physics, Harvard University, Cambridge, Massachusetts 02138, USA \\ ${ }^{3}$ Department of Physics, and Research Laboratory of Electronics, Massachusetts Institute of Technology, Cambridge, \\ Massachusetts 02139, USA
}

(Received 15 October 2019; revised manuscript received 10 February 2020; accepted 14 February 2020; published 30 March 2020)

\begin{abstract}
The frictional dynamics of interacting surfaces under forced translation are critically dependent on lattice commensurability. The highly nonlinear system of an elastic atomic chain sliding on an incommensurate periodic potential exhibits topological defects, known as kinks, that govern the frictional and translational dynamics. Performing experiments in a trapped-ion friction emulator, we observe two distinct structural and frictional phases: a commensurate high-friction phase where the ions stick-slip simultaneously over the lattice, and an incommensurate low-friction phase where the propagation of a kink breaks that simultaneity. We experimentally track the kink's propagation with atom-by-atom and sublattice site resolution and show that its velocity increases with commensurability. Our results elucidate the commensurate-incommensurate transition and the connection between the appearance of kinks and the reduction of friction in a finite system, with important consequences for controlling friction at nanocontacts.
\end{abstract}

DOI: 10.1103/PhysRevResearch.2.013380

\section{INTRODUCTION}

Commensurability at the interface between two atomically smooth, elastic surfaces can fundamentally alter the energetic cost of their forced relative motion [1,2]. Commensurate surfaces experience the largest sticking forces, and thus the most discontinuous form of motion: stick-slip friction [3]. At sufficient mismatch between the two surface lattices, the interface develops defects distributed over a collection of atoms-kink solitons-that result in an incommensurate phase with smoother surface translation, reduced energy barriers, and reduced friction [4]. Thus the appearance of kinks, that requires finite lattice mismatch, marks the commensurateincommensurate (C-I) transition [5-8].

In the solid state, two-dimensional versions of the C-I transition were observed at the interface between krypton monolayers and graphite [9], and between graphene and hexagonal boron nitride [10]. There, rotation between two surfaces introduced a lattice mismatch and the transition between a commensurate and an incommensurate phase. However, a direct experimental link between the C-I transition and the appearance of a finite kink density, supported by an atomistic calculation of the energy landscape [11], is challenging in such systems.

\footnotetext{
*dag50@cam.ac.uk

†vuletic@mit.edu

Published by the American Physical Society under the terms of the Creative Commons Attribution 4.0 International license. Further distribution of this work must maintain attribution to the author(s) and the published article's title, journal citation, and DOI.
}

Typically, the Frenkel-Kontorova model [12] is used to describe the physics of commensurability of extended interfaces. In this model, the motion of kinks occurs within a potential landscape, the Peierls-Nabarro (PN) potential. The concepts of the PN potential and kinks naturally extend to small systems, as for example in the diffusion of epitaxial islands and surface adsorbates [4,13-15]. Small systems offer an opportunity to study the formation of kinks under conditions where a direct link between concepts and experimental observations can be established.

Friction emulators with synthetic interfaces consisting of colloidal particles [16] or cold trapped ions [17] have improved our understanding of fundamental surface science, owing to in-situ tuning of fundamental parameters, and to the ability to image individual particles. Such emulators [18-20] have been used to observe the Aubry transition [21-23] and kink transport [16,24]. Cold trapped-ion systems [25-28] have been used to study fundamental aspects of atomistic friction, such as mismatch-induced lubricity [17], the temperature and velocity dependence of friction [29], and multislip friction [30].

In this paper, we use a trapped-ion friction emulator to experimentally observe a structural phase transition captured by the appearance of kinks: the few-atom analog of the C-I transition. We observe the stick-slip dynamics of the chain atom by atom, find that a critical degree of commensurability is required for kinks to form, and tie their appearance to a reduction in the observed friction. In our finite onedimensional system, a kink is a metastable configuration of the atoms $[4,36,37]$ - it has a higher energy than the global minimum with respect to translation - that is manifested in our experimental signal by atoms slipping at different times. 
(a)

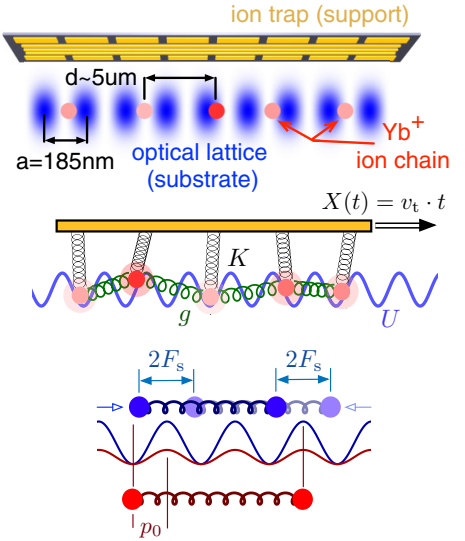

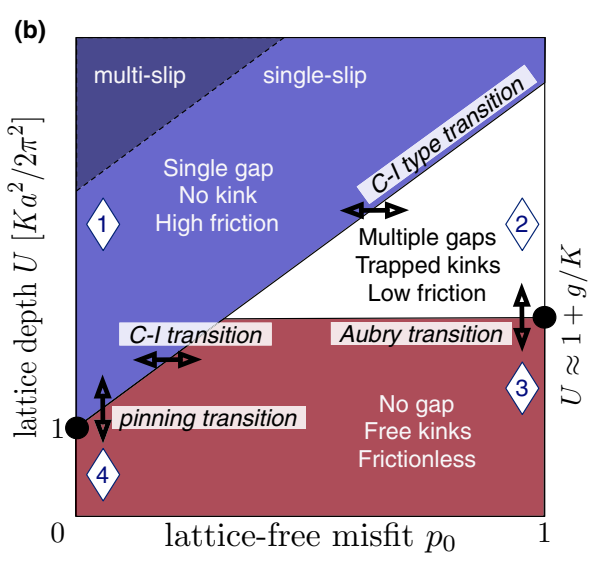

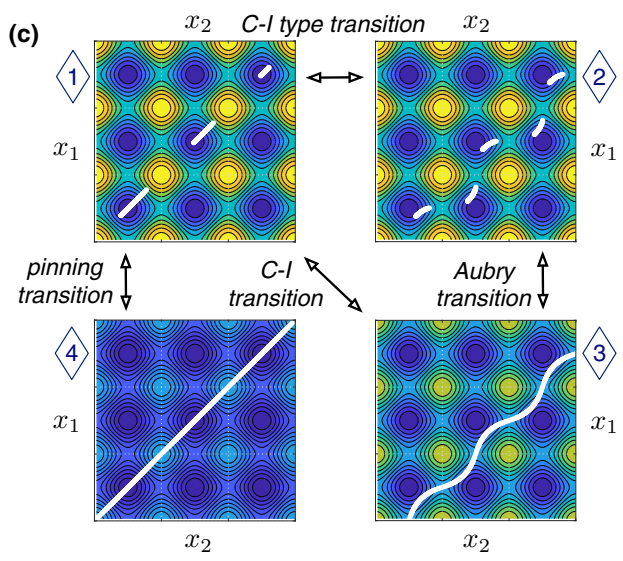

FIG. 1. Ion-crystal friction emulator for the study of structural phases and kinks. (a) Our friction emulator system [17,28,31] consisting of an ion chain in a Paul trap (support) with typical axial vibrational frequency $\omega_{0} /(2 \pi) \sim 360 \mathrm{kHz}$ and average interion spacing $d \sim 5 \mu \mathrm{m}$, subject to an optical-lattice potential (substrate) with depth $U / k_{B} \sim 1 \mathrm{mK}$ and lattice constant $a=185 \mathrm{~nm}$. The ions are laser-cooled into the optical lattice to temperatures $\gtrsim 0.05 U / k_{B}$. The crystal is displaced across the lattice with ion-trap electric fields. Each ion's position relative to the lattice is observed via the position-dependent ion fluorescence that results from lattice-assisted laser cooling [17], as the trap center $X$ is translated at velocity $v_{t}$. Each ion's interaction with the support and with its neighbor are captured by spring constants $K$ and $g$, respectively [21,32] (see Appendix H). The lattice-free misfit $p_{0}$ is measured at low lattice depth $U$ (red). At high lattice depth (blue), displacement hysteresis is equivalent to friction $F_{s}$. (b) Schematic structural phase diagram for a two-ion chain in the ( $\left.p_{0}, U\right)$ plane. Arrows highlight transitions of interest. (c) Adiabatic path (white curve) of a two-ion chain through the lattice energy landscape (minima in blue, maxima in yellow) as the trap is translated vs ion positions $x_{1}, x_{2}$, shown for points of interest in the $\left(p_{0}, U\right)$ plane.

\section{NANOFRICTION EMULATOR}

Our emulator [17,28,31] consists of a self-organized onedimensional Coulomb crystal of $N$ laser-cooled ${ }^{174} \mathrm{Yb}^{+}$ions in a linear Paul trap [33], sliding over a periodic optical potential of depth $U$ and lattice spacing $a=185 \mathrm{~nm}$ generated by a standing wave of light [Fig. 1(a)]. The Paul trap's vibrational frequency is tuned over the range $350-375 \mathrm{kHz}$, and the optical lattice depth is calibrated to be $U=19 \mathrm{MHz}$ (see Appendix C) for all experiments (except during calibration measurements of the lattice-free misfit $p_{0}$, during which it is set to $U \approx 4 \mathrm{MHz}$ ). A translation of the Paul trap's potential minimum $X$ with respect to the optical lattice transports the ion crystal at adjustable speed $v_{t}$ over the periodic potential. Continuous laser cooling of the ions turns the optical lattice potential that each ion feels into a proportional amount of fluorescence (see Appendix A). As a result, the position of each ion relative to the optical lattice is tracked with subwavelength resolution via their fluorescence, allowing us to measure each ion's hysteresis loops [17] and to reconstruct a kink traveling through the ion chain. Continuous laser cooling of the ion chain removes heat generated by friction, and sets an ion-chain temperature $T=0.05 U / k_{B}$ when measured in the absence of external drive. Meanwhile the effects of a finite temperature are reduced by performing experiments at sufficiently high translation speed [29], here fixed at $v_{t} / a=$ 2000 per second.

Although the intrinsic interion spacing $d_{j, j+1}$, from ion $j$ to ion $j+1(j=1, \ldots, N-1)$, of a few micrometers is not uniform along the chain, it can be controlled with nanometer precision by adjusting the Paul trap harmonic potential in order to introduce a misfit $p_{0}$ between the ion crystal and the lattice [Fig. 1(a)]. This is measured under translation of the ion chain over an optical lattice that only weakly affects the ion chain motion $\left(U \ll K a^{2} / 2 \pi^{2}\right)$, as follows (see also
Appendix B). We label $X_{j}^{(i)}$ the $i$ th position of the Paul trap minimum at which ion $j$ passes a lattice antinode during translation, which appears as a fluorescence maximum. We set our reference as $X_{1}^{(1)}=0$-i.e., we reference the position of all maxima to the edge ion first maximum — and we set our length unit as $X_{1}^{(2)}-X_{1}^{(1)}=1-$ i.e., we set the distance between two slips of the same ion, and therefore the lattice period, to be unity. A simultaneous measurement of each ion's fluorescence can thus be used to determine $X_{j}^{(i)}$ in these units. Because all ions necessarily pass a lattice antinode if the chain is translated by one lattice period $a, X_{j+1}^{(i)}-X_{j}^{(i)}$ is a direct measurement of the average distance between ion $j$ and ion $j+1$ relative to the lattice spacing: $\left(d_{j, j+1} / a\right) \bmod 1$. Averaging this quantity over the ion chain is our measure of the lattice-free misfit,

$$
p_{0} / 2=\frac{1}{N-1} \sum_{j=1}^{N-1}\left(d_{j, j+1} / a\right) \bmod 1 .
$$

The chain is in-registry with the substrate when each ion spacing $d_{j, j+1}$ is an integer multiple of the lattice spacing $a,\left(d_{j, j+1} / a\right) \bmod 1=0$, corresponding to no misfit, $p_{0}=$ 0 . A departure from this configuration introduces a misfit $p_{0}>0$ whose value can be tuned continuously up to its maximum $p_{0}=1$ for $N=2$, and up to $p_{0} \approx 0.8$ for $N=5$ (limited to this value owing to spacing inhomogeneities in the longer ion chain). Equation (1) is useful for quasiuniform phase spacing of the chain, i.e., $\mid\left[\left(d_{j+1, j+2} / a\right) \bmod 1\right]-$ $\left[\left(d_{j, j+1} / a\right) \bmod 1\right] \mid \ll 1$, and is valid for $0 \leqslant\left(d_{j, j+1} / a\right) \bmod$ $1 \leqslant 0.5$; beyond 0.5 , corresponding to the maximal misfit $p_{0}=1$, the system "wraps around" and the misfit decreases. A nonzero misfit can cause up to $N p_{0} / 2$ kinks, i.e., the kink density $p_{\mathrm{k}}$ is at most $p_{0}$ [5]. Here we tune the lattice-free misfit 
$p_{0}$ from 0 to 1 for $N=2$, and from 0 to 0.4 for $N=5$, which introduces at most one kink.

\section{STRUCTURAL PHASES}

Several structural phases are expected as a function of the lattice-free misfit $p_{0}$ and the lattice depth $U$, as shown schematically in Fig. 1(b). For large misfit $p_{0} \sim 1$, emulating the maximally incommensurate case for infinite chains, the Aubry transition [34] at critical lattice depth $U_{c}$ takes the system from a frictionless phase (for $U<U_{c}$ ) to a pinned phase (for $U>U_{c}$ ), as observed previously in our finite system [21]. By contrast, the C-I transition occurs as a function of the misfit $p_{0}$, and can be identified by the appearance of kinks [5].

Figure 1(c) illustrates these important transitions for a finite system in the limiting case $N=2$. For a sufficiently commensurate arrangement $p_{0} \sim 0$ [Fig. 1(b), diamond 1], as the trap position $X$ is translated over the lattice potential, the chain is pinned into a configuration where the atoms are in-registry with the lattice, and where the forced translation of the chain creates sudden transitions towards the same stable configuration shifted by one lattice site [Fig. 1(c), diamond 1]. The stick-slip events of all atoms in the chain are synchronous and result in the largest friction force-a regime well described by Prandtl-Tomlinson physics [1]. As the misfit $p_{0}$ is increased, the different atoms experience different lattice forces-a regime better described by FrenkelKontorova physics [12]. At a critical value $p_{0} \geqslant p_{c}(U)$, it becomes energetically favorable under forced translation for some of the atoms in the chain to slip to the next lattice site, while the rest of the chain remains in its initial lattice site [Fig. 1(c), diamond 2]. This event defines a configurational change in the chain to a new metastable state that contains a kink defect [4,35] [Fig. 1(b), diamond 2]. The appearance of a kink embodies a C-I transition [5] for a finite system, although the metastable kink state is not truly incommensurate $[4,36,37]$. In this configuration, the PN barriers are finite: kinks are trapped, the translation of the chain is still dominated by stick-slip events, and the adiabatic trajectory has gaps and is thus not analytic. Below a critical value of the lattice depth $\left(U<U_{c}\right)$, marking the Aubry transition, the PN barriers disappear: the movement of kinks is free, the translation of the chain over the substrate is continuous, the function defining its adiabatic trajectory is analytic, and friction disappears [Figs. 1(b) and 1(c), diamond 3]. PN barriers also disappear at $p_{0} \sim 0$ when the trap force exceeds the maximal substrate force [38], $U<K a^{2} / 2 \pi^{2}$ [Fig. 1(b), diamond 4].

\section{C-I TRANSITION, KINK DENSITY, AND FRICTION}

The kink density $p_{\mathrm{k}}$ is simply the misfit measured in the presence of the lattice [5], as it quantifies the deviation per particle from a commensurate arrangement. At a critical value of $p_{0}$ that depends on the lattice depth $U$, the kink density $p_{\mathrm{k}}$ undergoes a transition from $p_{\mathrm{k}}=0$ to $p_{\mathrm{k}}>0$, as shown in Fig. 2(a) for an infinite Frenkel-Kontorova chain. This critical point $p_{c}(U)$, beyond which $p_{\mathrm{k}}$ quickly converges to $p_{0}$, indicates the C-I transition [5], and this $p_{\mathrm{k}}$ versus $p_{0}$ parametrisation is precisely how we observe it.
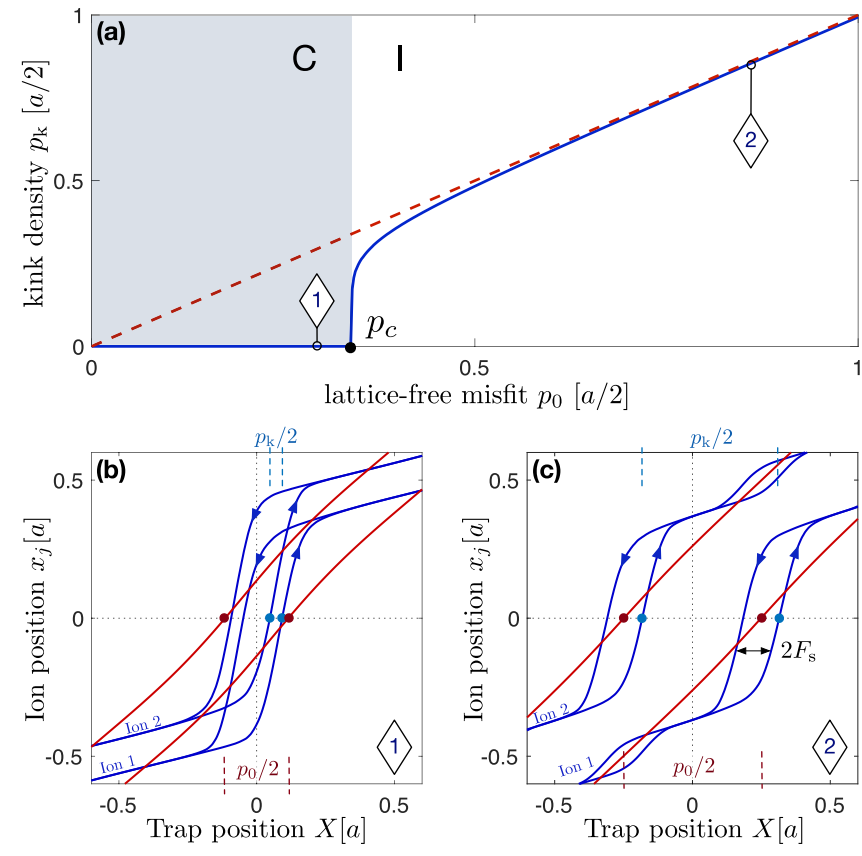

FIG. 2. The C-I transition via kink density and hysteresis. (a) Kink density $p_{\mathrm{k}}$ vs lattice-free misfit $p_{0}$ in the infinite onedimensional Frenkel-Kontorova model, showing the C-I transition [5] at the critical misfit $p_{c}$ separating the commensurate (C) and incommensurate (I) phases. (b-c) Simulated ion positions $x_{j}$ (mod a) vs trap position $X$, for two ions, in their lattice-free position (red) and lattice-perturbed position (blue), where forward and backward translation results in static friction $F_{s}$. The difference in trap positions at which the ions pass lattice maxima $x_{j}=0$ corresponds to the misfit $p_{0}$ (red) and the kink density $p_{\mathrm{k}}$ (blue). (b) $p_{0}=0.3$ and (c) 0.8 .

To observe this transition in our finite chain, we measure $p_{\mathrm{k}}$ in an identical way to $p_{0}$, albeit in the presence of a deep lattice $U=4.6 \mathrm{Ka}^{2} / 2 \pi^{2}$ : from fluorescence measurements we obtain the trap positions $X_{j}^{(i)}$ at which ion $j$, under forced translation, passes a lattice maximum $\left(x_{j}=0\right)$ in relation to its neighbor $j+1$. A difference $X_{j+1}^{(i)}-X_{j}^{(i)}$, leading to $p_{\mathrm{k}}>0$, captures whether slip events are asynchronous and therefore whether a kink is present. In the prototypical case $N=2$, Figs. 2(b) and 2(c) shows the simulated trajectories $x_{1}, x_{2}$ of two ions against trap position $X$ (see Appendix E for $N=5)$. The red curves are the lattice-free $(U=0)$ trajectories: the horizontal distance between them is the latticefree misfit $p_{0}$, and overlapping curves $\left(p_{0}=0\right)$ show the chain is commensurate with the lattice. The blue curves are trajectories in the presence of a deep lattice $\left(U>U_{c}\right)$ : they exhibit hysteresis-and therefore friction-corresponding to a gapped adiabatic trajectory [Fig. 1(c)]. At misfits below the C-I transition [Fig. 2(b)], the trajectory has a single gap (per lattice period), and the hysteresis loops of the two ions overlap almost completely. This corresponds to synchronous slip events characterized by a kink density $p_{\mathrm{k}} \sim 0$. At large misfit [Fig. 2(c)], the hysteresis loops exhibit no overlap, corresponding to asynchronous slip events and to the maximum possible kink density $p_{\mathrm{k}} \sim p_{0}$. The doubling of gaps (per lattice period) in the adiabatic trajectory, and the consequent doubling of hysteresis loops, can be understood as two transi- 


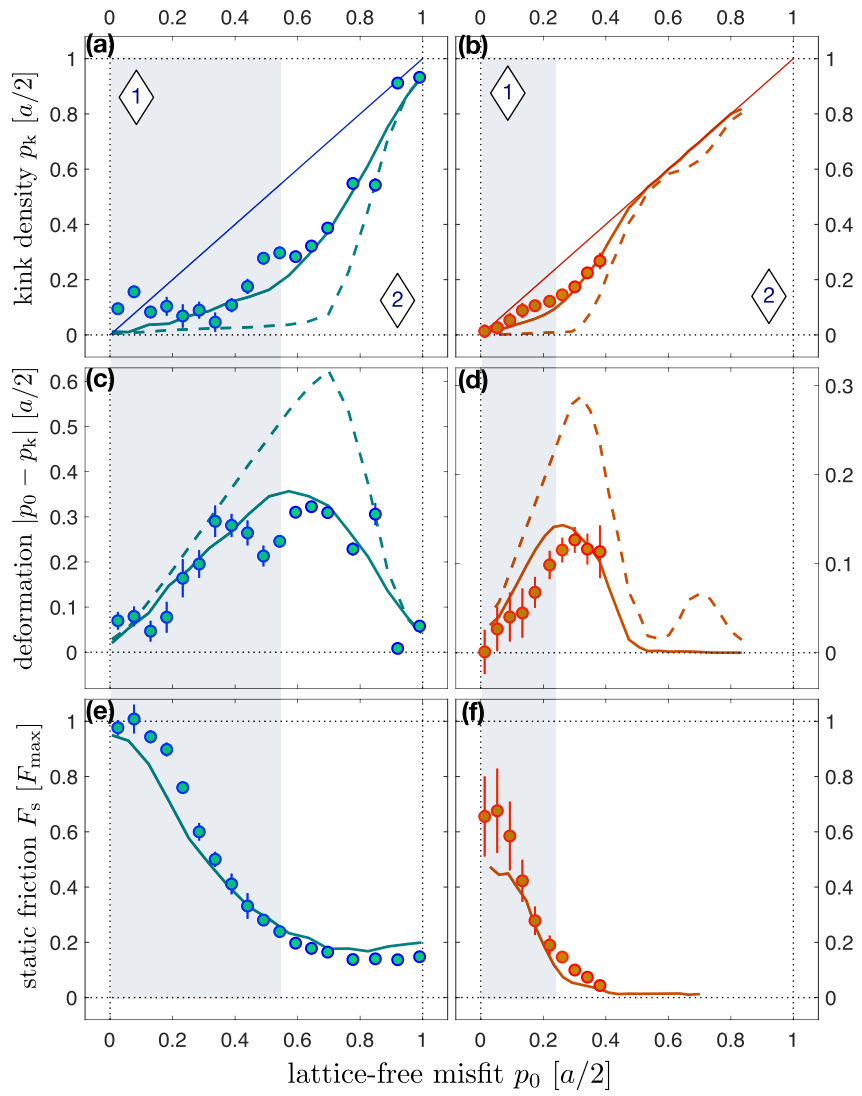

FIG. 3. Kinks in a two-ion chain (left) and a five-ion chain (right). Kink density $p_{\mathrm{k}}\left[(\mathrm{a})\right.$ and (b)], deformation $\left|p_{0}-p_{\mathrm{k}}\right|[(\mathrm{c})$ and (d)], and static friction force $F_{s}$ [(e) and (f)], measured vs lattice-free misfit $p_{0}$ for $N=2$ [blue, (a), (c), and (e)] and $N=5$ [red, (b), (d), and (f)]. Lines in (a) and (b) are the kink density $p_{\mathrm{k}}=p_{0}$ for $U=0$. Solid curves in (a)-(f) are numerical simulations for parameters matching those of the data shown, using $k_{B} T / U=0.2$ [(a), (c), and (e)] and 0.5 [(b), (d), and (f)]. Dashed curves are $T=0$ simulations. The critical misfit $p_{c}(U)$ is the misfit $p_{0}$ that maximizes $p_{0}-p_{\mathrm{k}}: p_{c}(U)=0.55(N=2)$ and $p_{c}(U)=0.25(N=$ 5). Numbered diamonds match Fig. 1 points of interest. $F_{\max }$ is the maximal friction in the single-slip regime $[29,30]$.

tions: the entry of a kink at one end of the chain, and its exit at the other end. The intermediate state is a distinct, metastable configuration of the chain containing a trapped kink [4].

Figure 3 shows the measured kink density $p_{\mathrm{k}}$ and static friction force $F_{s}$ as a function of the lattice-free misfit $p_{0}$ for a two- and a five-ion chain. The trap positions $X_{j}$ at which ion $j$ passes a lattice antinode (the "slip" positions) appear as fluorescence peaks in our experiment [17]. A linear fit of the slip positions $X_{j}$ versus $j$ gives $p_{\mathrm{k}}$, while the difference between forward and backward slips gives $F_{s}$. The lines $p_{\mathrm{k}}=$ $p_{0}$ in Figs. 3(a) and 3(b) represent the expected behavior for $U \ll U_{c}$, when ion movement is unperturbed by the lattice. A finite lattice depth $\left(U>U_{c}\right)$ pins the ions to lattice minima even for $p_{0}>0$, causing the ions to slip more synchronously, and thereby lowering the kink density to $p_{\mathrm{k}} \ll p_{0}$. Indeed, measurements of $p_{\mathrm{k}}$ as a function of $p_{0}$ agree qualitatively with the critical behavior expected from the infinite-chain Frenkel-Kontorova physics [Fig. 2(a)]: for $p_{0}$ less than a criti- cal value $p_{c}, p_{\mathrm{k}}$ stays close to 0 (synchronous slipping), while above that critical value, $p_{\mathrm{k}}$ ascends rapidly back towards $p_{0}$ (asynchronous slipping). The transition is highlighted by the deformation $\left|p_{0}-p_{\mathrm{k}}\right|$, which reaches a maximum at the critical point $p_{c}(U)$, as shown in Figs. 3(c) and 3(d).

The transition is smoother than expected from an infinite Frenkel-Kontorova chain, which we attribute to finitetemperature and finite-size effects. Simulations of a finite chain at temperature $T$ (see Appendix D), shown as solid curves in Figs. 3(a)-3(d), are in good agreement with our data using $k_{B} T / U=0.2$ for $N=2$ and $k_{B} T / U=0.5$ for $N=5$. By comparison, zero-temperature simulations of the same systems, shown as dashed curves, exhibit a sharper transition that occurs at a higher value of the misfit $p_{0}$. Temperature effectively reduces the PN barriers that cause stick-slip motion, making it more likely for asynchronous slips to occur, and thus increasing $p_{\mathrm{k}}$ relative to its zero-temperature value. The additional step structure for $N=5$ reflects the fact that, for $N>3$, intermediate values of the misfit correspond to the system entering higher-order commensurate phases. As $N$ grows the number of steps grows until, for $N=\infty$, this structure constitutes a Devil's staircase [5,12].

The transition points $p_{c} \sim 0.55$ for $N=2$ and $p_{c} \sim 0.25$ for $N=5$, associated with the appearance of kinks, also delineate two frictional phases, as shown in Figs. 3(e) and 3(f). In the region $p_{0} \lesssim p_{c}$ (Fig. 1 , diamond 1) where $p_{\mathrm{k}} \sim 0$ and the slips are synchronous, friction is high, while in the region $p_{0} \gtrsim p_{c}$ (Fig. 1, diamond 2 ) where $p_{\mathrm{k}} \sim p_{0}$ and the slips are asynchronous, friction is low. The transition point $p_{c}$ also marks a reduction in the dependence of friction $F_{s}$ on the misfit $p_{0}$, thus confirming that the presence of a kink defect changes the frictional response of the system.

\section{TRAVELING KINK}

The incommensurate phase $\left(p_{0}>p_{c}\right)$ defined by asynchronous slipping must exhibit a traveling kink, whose direct measurement is possible in a sufficiently long chain. We confirm this from the position of all ions in a five-ion chain as they traverse the optical lattice. Using their known positiondependent fluorescence across the lattice, which follows a $\cos ^{4}\left(\pi x_{j}\right)$ function, we reconstruct each ion's position from its fluorescence [39]. We obtain pairwise the neighbor distance $\Delta x_{j, j+1}(j=1, \ldots, 4)$, and track a compression and extension in relative position within each ion pair as the background trap is translated [Fig. 4(a)]. Grey shading denotes low fluorescence, and therefore limited reconstruction fidelity (see Appendix G).

Clear oscillations in the neighbor distances [Fig. 4(a)] reveal a density wave traveling through the discrete system: a kink enters the chain at its free end, travels through it and exits at the other free end, causing a staggered signal across pairs which repeats at the lattice period $a$ as the Paul trap translates the chain. For a given pair, the distance $\Delta x_{j, j+1}$ reaches a maximum when the kink profile is centered on that pair. This analysis is supported by finite-temperature numerical simulations of our experiment with a five-ion chain [Figs. 4(b) and 4(c)]. In Fig. 4(b), the signal $\Delta x_{j, j+1}$ is reconstructed from simulated fluorescence curves, processed identically with our data. In Fig. 4(c), $\Delta x_{j, j+1}$ is calculated from the simulated 

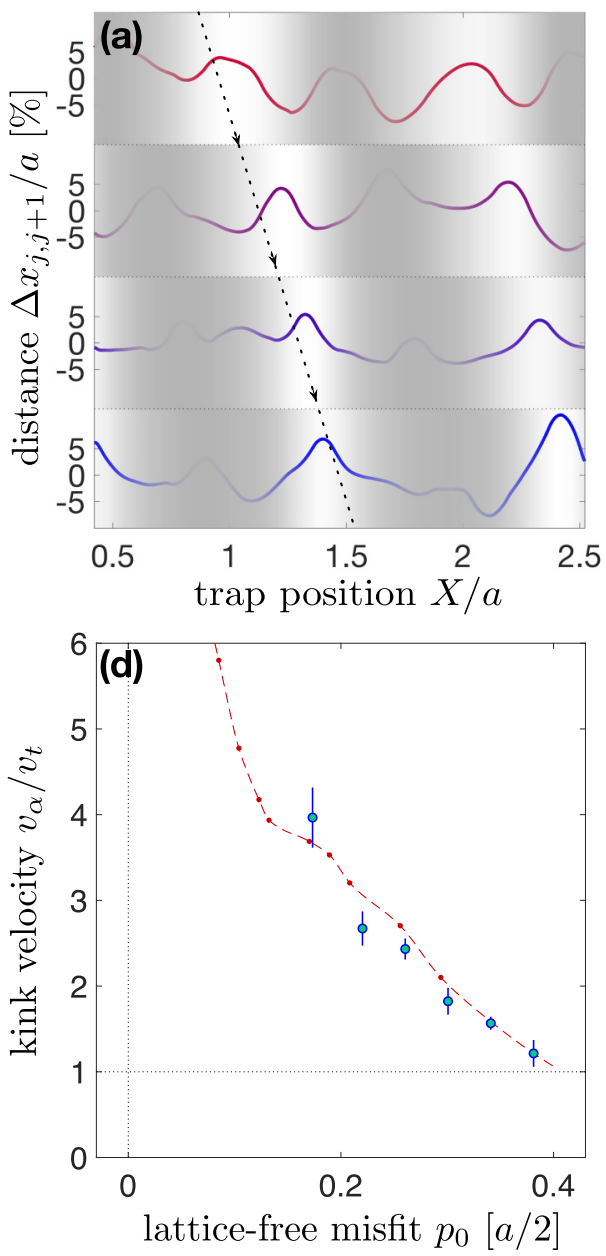
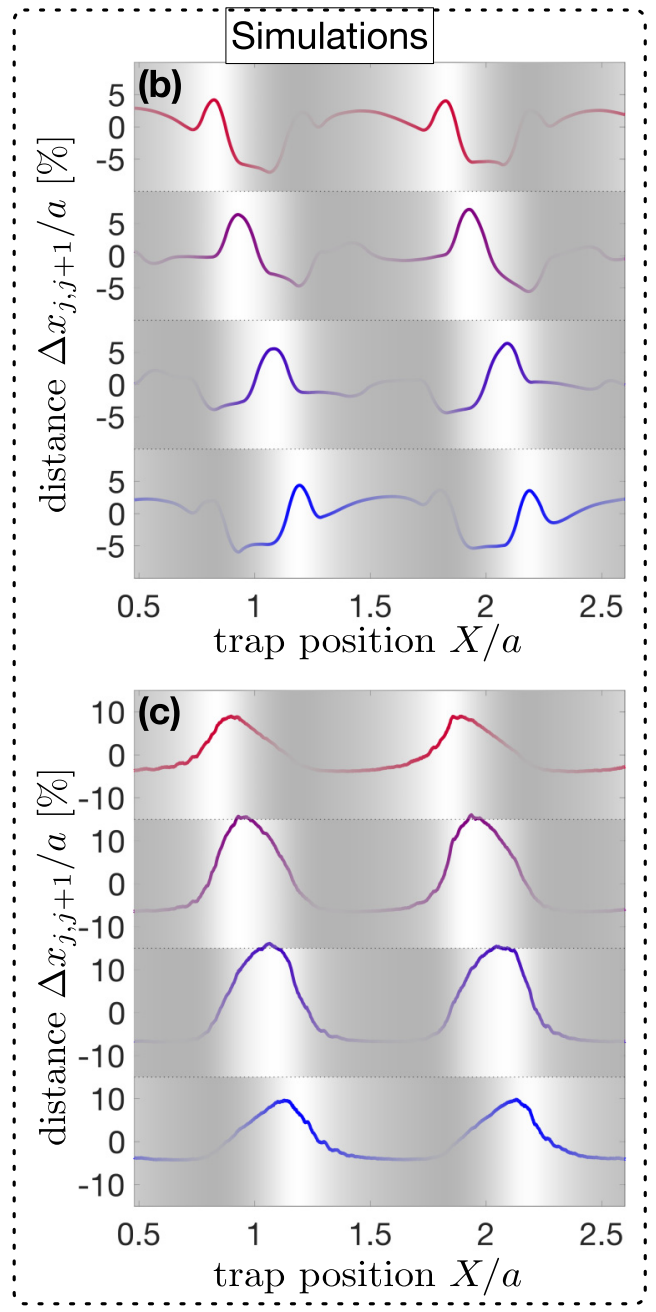

FIG. 4. Propagation of a kink in a five-ion chain (a) The distance $\left(\Delta x_{j, j+1}=\left(x_{j}-x_{j+1}\right)\right)(\bmod a)$ between ion $j$ and its nearest neighbor $j+1$ (with mean subtracted) vs trap position $X$. From top to botttom, $j=1, \ldots, 4$. Dashed line highlights propagation of the kink maximum across the ion pairs. Grey shading indicates regions where fluorescence is low, and position is extracted with lower confidence. (b) Numerical simulations for parameters matching those of data in (a). $\Delta x_{j, j+1}$ is reconstructed from a simulated fluorescence signal. Grey shading indicates regions where simulated fluorescence is low. (c) $\Delta x_{j, j+1}$ from simulated position curves (see Appendix G). (d) Kink velocity $v_{\alpha}$, normalized by trap velocity $v_{t}$, against lattice-free misfit $p_{0}$. The dashed line is from simulation.

position curves-which, unlike in the experiments, is directly accessible in simulation. While the curves reconstructed from fluorescence [Fig. 4(b)] are clearly deformed, and exhibit the same erroneous deformations as our data in the lowfluorescence regions (grey area), they are a good approximation to the true distance [Fig. 4(c)]. Although the true distance [Fig. 4(c)] is accessible in simulations independent of the fluorescence level, we apply grey shading to highlight that the true kink deformations are indeed centered on regions of high fluorescence.

Tracking the positions of the kink maximum across the pairs as the trap is translated [Fig. 4(a)] yields the velocity $v_{\alpha}$ of the traveling kink. This is summarized in Fig. 4(d), where the kink velocity is normalized by the trap translation velocity $v_{t}=d X / d t$. The dashed curve is obtained from our numerical simulations of the fluorescence signal. Our data reproduce the salient feature in the simulation: the kink velocity increases with decreasing misfit $p_{0}$ as expected from a system approaching a commensurate phase with increasingly synchronous slips. Below $p_{0} \approx 0.18$, the staggered kink signal fades in both data and simulation, in agreement with a transition to the commensurate phase.

\section{SUMMARY AND OUTLOOK}

In summary, we observe atom-by-atom the appearance of a kink in a finite system and connect it to a structural phase transition between a commensurate phase, where friction is high, and an incommensurate phase, where friction is reduced. This work could enable the study of interacting topological defects and frustration at a nanocontact [12]. Control over the atomic configuration of a chain yields detailed insight into the stick-slip dynamics of a commensurate versus an incommensurate phase, building intuition that could carry over to experiments with, for example, two-dimensional heterostructures. Furthermore, quantum tunneling of ions through lattice barriers, in principle realizable in our system [39-41], could 
introduce a quantum-mechanical picture of kinks with relevance at the nanoscale and at cold surfaces.

\section{ACKNOWLEDGMENTS}

This work was supported in part by the NSF, the NSF CUA, and the MURI program through ONR. D.A.G. acknowledges support from a St John's College (Cambridge) Title A Research Fellowship.

\section{APPENDIX A: POSITION DETECTION VIA FLUORESCENCE}

Our dynamic position curves $x_{j}$ are reconstructed from the observed ion fluorescence, which varies proportionally to the optical potential energy $U \cos ^{4}\left(\pi x_{j}\right)$ experienced by the ion. This is a result of our laser-cooling scheme, which uses the optical lattice to couple the vibrational levels $n$ and $n-2$ of the ion's quantized motion in the optical lattice well $[39,40]$. The spatial dependence of this Raman coupling is such that the off-resonant transition $n \rightarrow n$, which on resonance would be stronger by two orders of the Lamb-Dicke factor (which is $\approx 10 \%$ for our system), increases from lattice node to lattice anti-node proportionally to the optical potential. The stronger this coupling is, the larger the scattered fluorescence, resulting in the position-dependent fluorescence signal, which, when time-resolved, amounts to subwavelength imaging of the ion's average trajectory.

\section{APPENDIX B: EXTRACTING $p_{0}$ AND $p_{\mathrm{k}}$ FROM ION FLUORESCENCE TRACES}

In Fig. 5, we show example fluorescence data, taken from five ions in a five-ion chain. We collect the fluorescence pairwise (ion 1 and ion 2, ion 2 and ion 3, etc.) on a pair of photomultiplier tubes. The horizontal axis represents the fraction of our experiment's repetition time; each point is an average over many repetitions of identical translations of the chain, back and forth, over several lattice sites at a frequency defined by our external drive (which controls the translation velocity $[39,40])$. By syncing the time axes for commonly imaged ions (ion 2, 3, and 4 are imaged twice), we synchronize imaging for the entire chain. As described in the main text: we label $X_{j}^{(i)}$ the $i$ th position of the Paul trap minimum at which ion $j$ passes a lattice antinode during translation, which appears as a fluorescence maximum. We set our reference as $X_{1}^{(1)}=0$-i.e., we reference the position of all maxima to ion 1's first maximum-and we set our length unit as $X_{1}^{(2)}-X_{1}^{(1)}=1-$ i.e., we set the distance between two slips

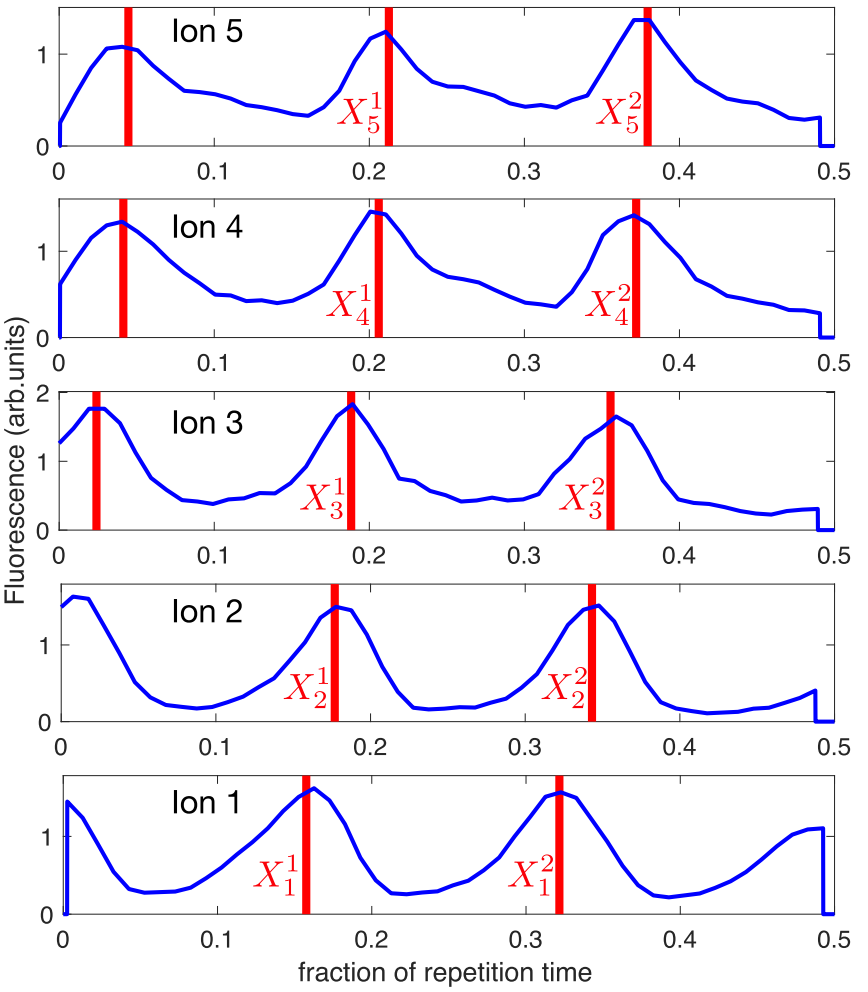

FIG. 5. Five-ion fluorescence traces. Experimental fluorescence data from five ions simultaneously imaged as they are translated through an optical lattice of depth $2 \pi^{2} U / K a^{2}=4.6$. Red vertical lines indicate the positions of fluorescence peaks, found as the maxima of parabolic fits of five data points near the maximum fluorescence point. These data yield $p_{\mathrm{k}}=0.17$.

and therefore the lattice period to be unity. A simultaneous measurement of each ion's fluorescence can thus be used to determine $X_{j}^{(i)}$ in these units. Because all ions necessarily pass a lattice antinode if the chain is translated by one lattice period $a, X_{j+1}^{(i)}-X_{j}^{(i)}(>0)$ is a direct measurement of the average distance between ion $j$ and ion $j+1$ relative to the lattice spacing: $\left(d_{j, j+1} / a\right) \bmod 1$.

In the main text, we provided an expression for $p_{0}$ which is valid for $\left(d_{j, j+1} / a\right) \bmod 1 \leqslant 0.5$, which for a quasiuniform chain (in mod space) is the largest possible misfit. Beyond, the periodicity of the optical lattice ensures that the total misfit decreases. For example, take the case where $\left(d_{j, j+1} / a\right) \bmod$ $1=0.99$; there, nearby atoms experience very similar lattice forces, and the misfit on a small chain is clearly close to 0 . Let us provide here a more complete expression for $p_{0}$ which can be used for all values of $\left(d_{j, j+1} / a\right) \bmod 1$ :

$$
p_{0} / 2=\frac{1}{N-1} \sum_{j=1}^{N-1} f\left(d_{j, j+1}\right)
$$

where

$$
f\left(d_{j, j+1}\right)=\left\{\begin{array}{lll}
\left(d_{j, j+1} / a\right) \bmod 1 & \text { for } & 0 \leqslant\left(d_{j, j+1} / a\right) \bmod 1<0.5 \\
1-\left(d_{j, j+1} / a\right) \bmod 1 & \text { for } \quad 0.5 \leqslant\left(d_{j, j+1} / a\right) \bmod 1<1
\end{array} .\right.
$$


At very low lattice depth $2 \pi^{2} U / K a^{2} \ll 1$, where the motion of the ions is nearly unaffected by the lattice, the procedure described above is used to obtain $p_{0}$. At large lattice depth $2 \pi^{2} U / K a^{2}=4.6$, such as in Fig. 5, and where we perform our measurements of the kink motion and kink misfit, this procedure yields $p_{\mathrm{k}}$.

\section{APPENDIX C: MEASUREMENT OF THE OPTICAL LATTICE DEPTH}

From the single-atom Prandtl-Tomlinson model [1], we know that for $2 \pi^{2} U / K a^{2}=4.6$ we obtain the largest friction force in a bistable potential. There the position hysteresis between forward and backward translation of an ion in the optical lattice reaches a value of $a$ exactly [39,40], meaning the forward and backward fluorescence signals are delayed by exactly one lattice spacing. This is because the metastable well in which the ion sits just before a slip event goes critical just when the harmonic trap sits above the next well a distance $a$ over. There, the friction force takes on the maximum value allowed in the so-called single slip regime [30], $F_{s}=K a / 2 \equiv$ $F_{\max }$. Measuring this for a single ion allows for our most precise calibration of the normalized lattice-depth parameter $\eta_{k}=2 \pi^{2} U / K a^{2}$ and therefore of the lattice depth $U$. Since $K=m \omega_{0}^{2}$ and $a$ are also known very precisely and independently, this provides an independent measurement of our lattice depth.

\section{APPENDIX D: NUMERICAL SIMULATIONS WITH LANGEVIN FORMALISM}

We follow standard numerical methods $[39,42,43]$ for calculating the mean position of a single particle in a periodic potential under the influence of an external shear force and a fluctuating force. We calculate the average trajectory $\left\langle x_{j}(t)\right\rangle$ of each particle $j$-cycling through all particles at each integration time step-over multiple integrations of the Langevin equation of motion:

$$
\begin{gathered}
m \ddot{x}_{j}+2 m \gamma \dot{x}_{j}+K\left(x_{j}-v_{t} t\right)+\frac{\pi U}{a} \cos \left(\frac{\pi x_{j}}{a}\right) \\
-\sum_{i \neq j} \frac{e^{2}}{4 \pi \epsilon_{0}} \frac{x_{j}-x_{i}}{\left|x_{j}-x_{i}\right|^{3}}=\xi_{j}(t),
\end{gathered}
$$

where the fluctuating force $\xi_{j}(t)$ satisfies the fluctuationdissipation theorem which relates its magnitude to the temperature $T$ and damping coefficient $2 m \gamma:\left\langle\xi_{j}(t) \xi_{j}\left(t^{\prime}\right)\right\rangle=$ $4 m \gamma k_{B} T \delta\left(t-t^{\prime}\right)$. We have taken $m=2.88934 \times 10^{-25} \mathrm{~kg}$ (the mass of a ${ }^{174} \mathrm{Yb}^{+}$ion), $\gamma=2 \times 10^{4} \mathrm{~s}^{-1}$ [29], and physical constants: electron charge $e$, vacuum permittivity $\epsilon_{0}$, and Boltzmann constant $k_{B}$.

The friction force is the value of the external force on the particle when it slips. The mean kink density $p_{\mathrm{k}}$, deformation $\left|p_{\mathrm{k}}-p_{0}\right|$, and friction force $F_{s}$ presented in the simulation curves throughout this paper are the values at the mean slipping time of the particle, as determined from the average trajectory $\left\langle x_{j}(t)\right\rangle$.

For all presented simulation curves, we average 150 instances of the simulation of forward and backward translation in the presence of the stochastic Langevin force. This results
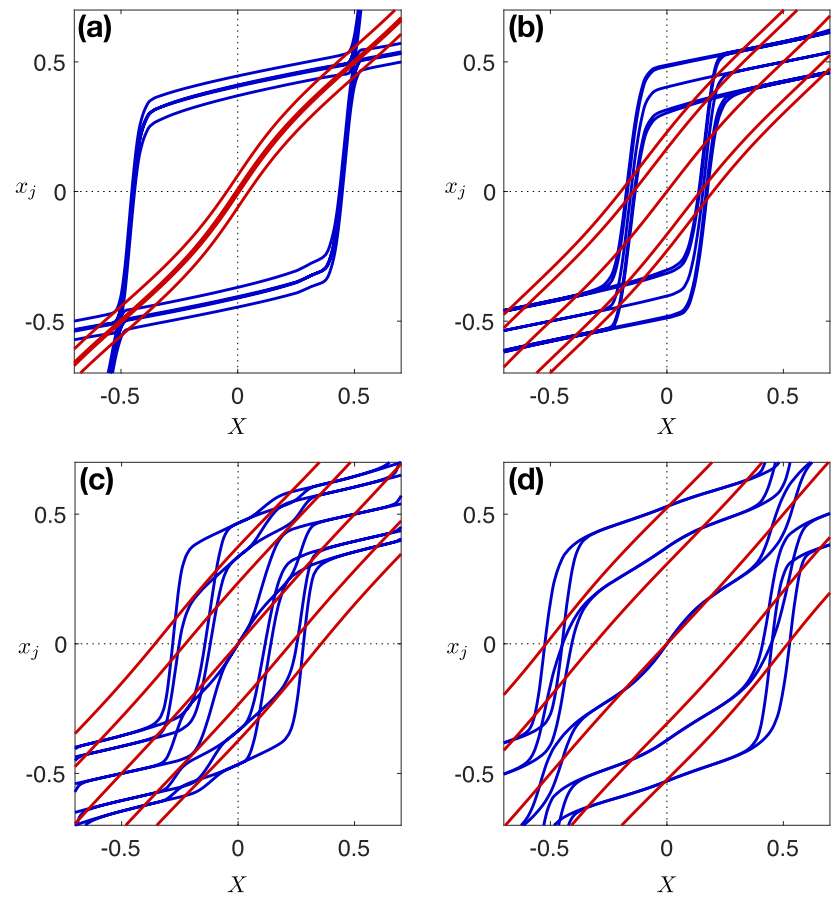

FIG. 6. Five-ion hysteresis loops for multiple misfits. Simulated hysteresis loops of ion positions $x_{j}$ vs trap position $X$, in their unperturbed position (red) and lattice-perturbed position (blue) as they are translated forward and backward by the trap, for $p_{0}=0$ (a), 0.26 (b), 0.38 (c), and $0.51(\mathrm{~d})$.

in a typical standard deviation at each ion position of $0.008 a$, which is negligible compared to kink feature sizes on the order of $0.1 a$ (see Fig. 4).

\section{APPENDIX E: APPEARANCE OF SECONDARY HYSTERESIS LOOP FOR $N=5$}

As shown in Fig. 2 of the main text for $N=2$, the increase in the kink signal $p_{\mathrm{k}}$ at the critical point $p_{c}$ is accompanied by the appearance of secondary hysteresis loops. In Fig. 6, we show simulations of hysteresis loops for $N=5$, and $k_{B} T / U=$ 0.05 , as the misfit $p_{0}$ is increased. We see the same doubling of hysteresis loops per lattice period as for $N=2$, i.e. the appearance of a kink state, occurring here around $p_{0} \sim 0.3$.

\section{APPENDIX F: DEPENDENCE OF C-I TRANSITION POINT ON $N$ AND $U$}

We show the simulated dependence of our kink deformation signal $\left|p_{\mathrm{k}}-p_{0}\right|$ as a function of $N$ [Fig. 7(a)] and $U$ [Fig. 7(b)], demonstrating that the transition pointassociated with the appearance of a single kink-depends on the length of the chain $N$ and the lattice depth $U$. A longer chain requires a smaller misfit to introduce a kink, while a larger lattice depth requires a larger misfit.

\section{APPENDIX G: KINK RECONSTRUCTION AND NUMERICAL SIMULATIONS}

Reconstructing the dynamic position curves $x_{j}$-used to get the neighbor distance $\Delta x_{j, j+1}$-from fluorescence traces 

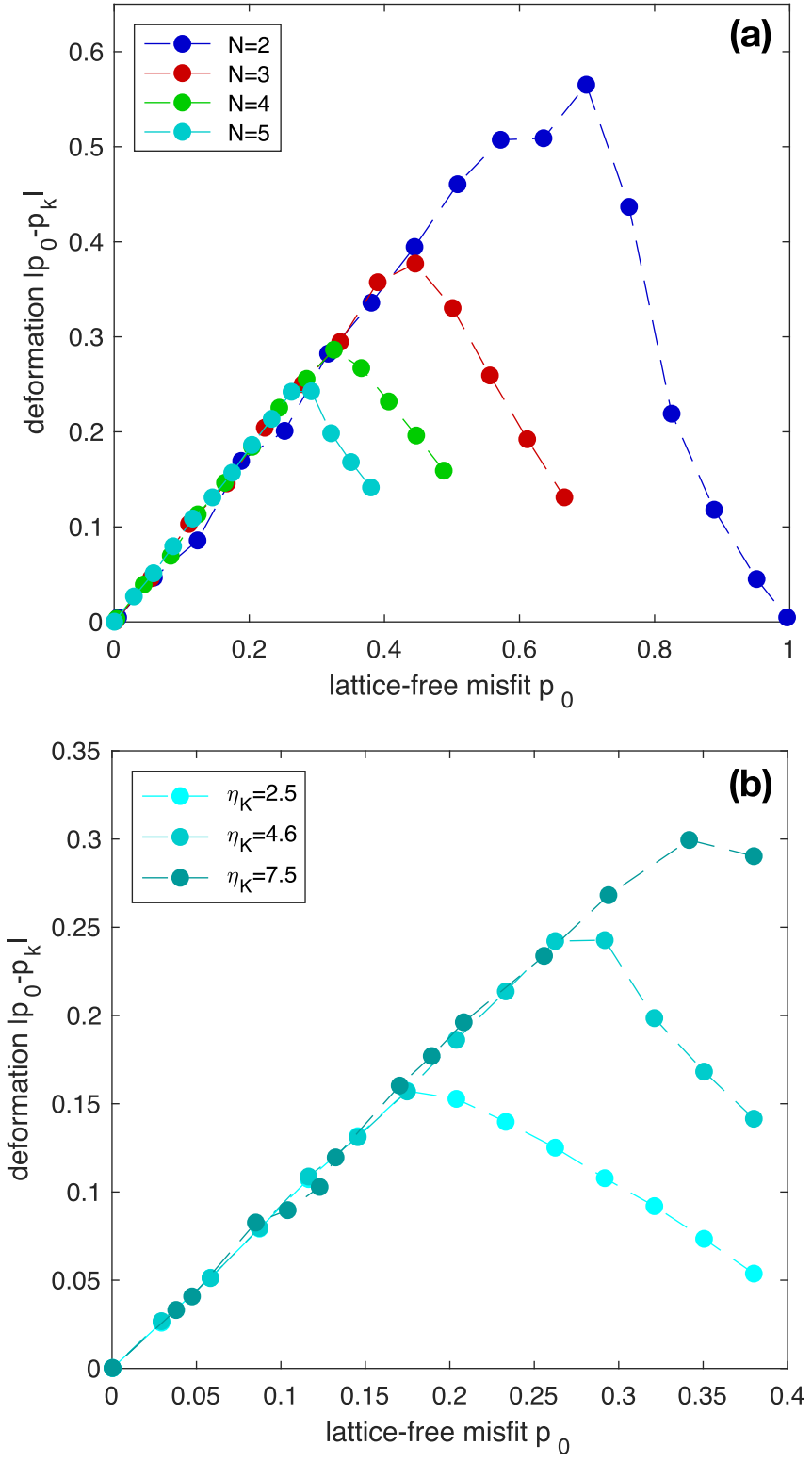

FIG. 7. The C-I transition as a function of $N$ and $U$. (a) Simulated kink deformation signal $\left|p_{\mathrm{k}}-p_{0}\right|$ at $\eta_{k}=2 \pi^{2} U / K a^{2}=4.6$ for $N=2,3,4,5$; and (b) at $N=5$ for $\eta_{k}=2 \pi^{2} U / K a^{2}=2.5,4.6,7.5$.

requires a few processing steps. Firstly, it is assumed that fluorescence maxima correspond to ions sitting at lattice antinodes. This is true when the friction force is 0 (i.e., there is no hysteresis, and all lattice positions are therefore allowed), and it is a good approximation when the friction is low, which is indeed the case in the incommensurate phase where kinks can be detected. Secondly, as mentioned in the main text, the fluorescence signal is insensitive to position around the lattice nodes because of the $\cos ^{4}\left(\pi x_{j}\right)$ dependence, and because of finite ion temperature which further averages the positiondependence in that region. This results in a fixed fluorescence signal around the nodes which makes it appear as though the ion is not moving. This in turn creates a signal resembling a compression, and thus a kink, when looking at the position difference with another ion whose fluorescence signal is
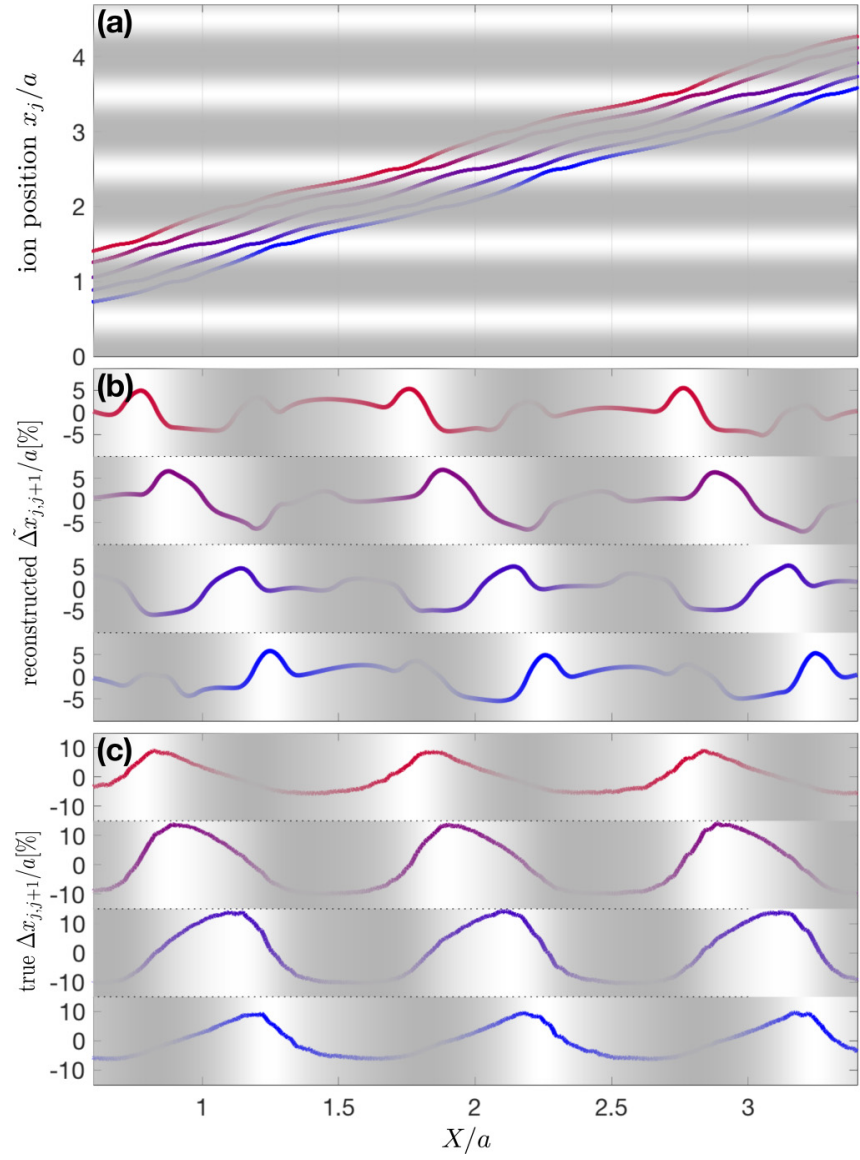

FIG. 8. Langevin numerical simulation of kinks in a five-ion chain. (a) The ion position $x_{j}$ of ion $j$ reconstructed from simulated fluorescence traces, expressed in units of lattice spacing $a$, as a function of the support position $X$. From red to blue, $j=1, \ldots, 5$. Grey shading indicates regions where simulated fluorescence is low, and therefore position is extracted with lower confidence. (b) The reconstructed distance $\tilde{\Delta x_{j, j+1}}=\left(x_{j}-x_{j+1}\right)$ between ion $i$ and its nearest neighbor $i+1$ (with mean subtracted), from positions $x_{j}$ shown in (a), expressed in units of lattice spacing $a$, as a function of the support position $X$. From red to blue, $j=1, \ldots, 4$. (c) The true distance between ion $j$ and its nearest neighbor $j+1, \Delta x_{j, j+1}=$ $\left(x_{j}-x_{j+1}\right)$, directly from the integrated equations of motion.

delayed (owing to asynchronous motion). This lower confidence in the reconstructed position of each ion is highlighted by grey shading in Fig. 4(a), representing trap positions where the average fluorescence of a pair of ions is low. By contrast, fluorescence is very sensitive to position on the slope of the lattice potential, and there temperature only plays a small role fractionally. This is why we can ignore the fluorescence information around the nodes, while using the fluorescence information elsewhere.

To further support this procedure, it is replicated identically on simulated fluorescence curves for which the true position information is available. Using the Langevin formalism (see Appendix D), we obtain finite-temperature average trajectories for each ion in a five-ion chain, as well as average fluorescence for each ion. Shown in Fig. 8(a) are the five position curves reconstructed from the simulated fluorescence curves. Shown in Fig. 8(b) is the same as Fig. 4(b): the four 
pair position difference curves calculated from the simulated reconstructed position curves, showing the kinks propagating from pair-to-pair. Shown in Fig. 8(c) is the same as Fig. 4(c): the four pair position difference curves calculated directly from the simulated position curves (these, evidently, are not available in experiments), also showing kinks propagating from pair-to-pair. All three panels also show as grey shading sections of the curves where the simulated fluorescence signal is low, and where therefore position reconstruction is expected to be inaccurate. While the reconstructed position and direct position curves in Figs. 8(b) and 8(c) differ in their exact shape, the qualitative features of staggered order across pairs, peak position, and skewness are replicated.

\section{APPENDIX H: COULOMB FORCES AS NEXT-NEIGHBOR SPRING FORCES}

This section explains the correspondence between our trapped-ion system and the Frenkel-Kontorova-Tomlinson model [21,32]. The harmonic confinement by external springs $K$ is provided by the axial confinement in the linear Paul trap at angular vibrational frequency $\omega_{0}=\sqrt{K / m}$, which corresponds to the center-of-mass (COM) motion of the chain. We take the interatomic springs $g$ to correspond to the highest- energy mode of the chain $\left(\omega_{\max }\right)$ - this is the mode for which the next neighbors near the center of the chain move in anti-phase. This gives the interatomic spring stiffness as $g \approx$ $\frac{1}{4} m \omega_{\max }^{2}$. We show here why this is a valid approximation.

Consider a small displacement $\delta x$ of one of the ions in the chain comparable to the lattice constant $a$. This is small compared to the equilibrium spacing of the ions in the Paul trap, $\delta x \leqslant a \ll d_{i j}$ where $d_{i j}$ is the distance between any two ions $i$ and $j$ in the chain. One can then approximate the neighbor spring forces by linearizing the Coulomb forces around the equilibrium distance:

$$
\delta F_{i j} \approx-2 \frac{e^{2}}{4 \pi \epsilon_{0}\left|d_{i j}\right|^{3}} \delta x .
$$

This gives the effective neighbor spring constant constant $g_{i j}=e^{2} / 2 \pi \epsilon_{0}\left|d_{i j}\right|^{3}$. From this expression, we can show that next-neighbor interactions dominate the mean-field interaction of a single ion with the rest of the chain, even for an infinite homogeneous chain: the sum of forces from farther neighbors, as a fraction of the nearest neighbor forces, is $\sum_{n=2, \infty} \frac{1}{n^{3}}=\zeta(3)-1 \approx 20 \%$ (where $\zeta$ is the Riemann zeta function).
[1] A. Vanossi, N. Manini, M. Urbakh, S. Zapperi, and E. Tosatti, Colloquium: Modeling friction: From nanoscale to mesoscale, Rev. Mod. Phys. 85, 529 (2013).

[2] S. Y. Krylov and J. W. M. Frenken, The physics of atomic-scale friction: Basic considerations and open questions, Phys. Status Solidi 251, 711 (2014).

[3] M. Urbakh, J. Klafter, D. Gourdon, and J. Israelachvili, The nonlinear nature of friction, Nature (London) 430, 525 (2004).

[4] O. M. Braun, Adiabatic motion of an atomic chain in periodic potential, Surf. Sci. 230, 262 (1990).

[5] P. Bak, Commensurate phases, incommensurate phases and the devil's staircase, Rep. Prog. Phys. 45, 587 (1982).

[6] V. L. Pokrovsky and A. L. Talapov, Ground State, Spectrum, and Phase Diagram of Two-Dimensional Incommensurate Crystals, Phys. Rev. Lett. 42, 65 (1979).

[7] S. N. Coppersmith, D. S. Fisher, B. I. Halperin, P. A. Lee, and W. F. Brinkman, Dislocations and the commensurateincommensurate transition in two dimensions, Phys. Rev. B 25, 349 (1982).

[8] H. P. Büchler, G. Blatter, and W. Zwerger, CommensurateIncommensurate Transition of Cold Atoms in an Optical Lattice, Phys. Rev. Lett. 90, 130401 (2003).

[9] S. C. Fain, M. D. Chinn, and R. D. Diehl, Commensurateincommensurate transition of solid krypton monolayers on graphite, Phys. Rev. B 21, 4170 (1980).

[10] C. R. Woods, L. Britnell, A. Eckmann, R. S. Ma, J. C. Lu, H. M. Guo, X. Lin, G. L. Yu, Y. Cao, R. V. Gorbachev, A. V. Kretinin, J. Park, L. A. Ponomarenko, M. I. Katsnelson, Y. N. Gornostyrev, K. Watanabe, T. Taniguchi, C. Casiraghi, H.-J. Gao, A. K. Geim, and K. S. Novoselov, Commensurateincommensurate transition in graphene on hexagonal boron nitride, Nat. Phys. 10, 451 (2014).
[11] L. Dezerald, L. Ventelon, E. Clouet, C. Denoual, D. Rodney, and F. Willaime, $A b$ initio modeling of the two-dimensional energy landscape of screw dislocations in bcc transition metals, Phys. Rev. B 89, 024104 (2014).

[12] O. M. Braun and Y. Kivshar, The Frenkel-Kontorova Model:2 Concepts, Methods, and Applications (Springer-Verlag, Berlin, Heidelberg, 2004).

[13] I. Markov and V. D. Karaivanov, A one-dimensional model for the mobility of small epitaxial islands II: Sinusoidal potential, Thin Solid Films 65, 361 (1980).

[14] C. Willis, M. El-Batanouny, and P. Stancioff, Sine-Gordon kinks on a discrete lattice. I. Hamiltonian formalism, Phys. Rev. B 33, 1904 (1986).

[15] A. Kwaśniewski, P. Machnikowski, and P. Magnuszewski, Kink dynamics in finite discrete sine-Gordon chains, Phys. Rev. E 59, 2347 (1999).

[16] T. Bohlein, J. Mikhael, and C. Bechinger, Observation of kinks and antikinks in colloidal monolayers driven across ordered surfaces, Nat. Mater. 11, 126 (2012).

[17] A. Bylinskii, D. Gangloff, and V. Vuletic, Tuning friction atomby-atom in an ion-crystal simulator, Science 348, 1115 (2015).

[18] A. Benassi, A. Vanossi, and E. Tosatti, Nanofriction in cold ion traps, Nat. Commun. 2, 236 (2011).

[19] D. Mandelli, A. Vanossi, and E. Tosatti, Stick-slip nanofriction in trapped cold ion chains, Phys. Rev. B 87, 195418 (2013).

[20] D. Mandelli, A. Vanossi, M. Invernizzi, S. Paronuzzi, N. Manini, and E. Tosatti, Superlubric-pinned transition in sliding incommensurate colloidal monolayers, Phys. Rev. B 92, 134306 (2015).

[21] A. Bylinskii, D. Gangloff, I. Counts, and V. Vuletić, Observation of Aubry-type transition in finite atom chains via friction, Nat. Mater. 15, 717 (2016). 
[22] J. Kiethe, R. Nigmatullin, D. Kalincev, T. Schmirander, and T. E. Mehlstäubler, Probing nanofriction and Aubry-type signatures in a finite self-organized system, Nat. Commun. 8, 15364 (2017).

[23] T. Brazda, A. Silva, N. Manini, A. Vanossi, R. Guerra, E. Tosatti, and C. Bechinger, Experimental Observation of the Aubry Transition in Two-Dimensional Colloidal Monolayers, Phys. Rev. X 8, 011050 (2018).

[24] J. Brox, P. Kiefer, M. Bujak, T. Schaetz, and H. Landa, Spectroscopy and Directed Transport of Topological Solitons in Crystals of Trapped Ions, Phys. Rev. Lett. 119, 153602 (2017).

[25] I. Bloch, Ultracold quantum gases in optical lattices, Nat. Phys. 1, 23 (2005).

[26] M. Enderlein, T. Huber, C. Schneider, and T. Schaetz, Single Ions Trapped in a One-Dimensional Optical Lattice, Phys. Rev. Lett. 109, 233004 (2012).

[27] R. B. Linnet, I. D. Leroux, M. Marciante, A. Dantan, and M. Drewsen, Pinning an Ion with an Intracavity Optical Lattice, Phys. Rev. Lett. 109, 233005 (2012).

[28] L. Karpa, A. Bylinskii, D. Gangloff, M. Cetina, and V. Vuletić, Suppression of Ion Transport due to Long-Lived Subwavelength Localization by an Optical Lattice, Phys. Rev. Lett. 111, 163002 (2013).

[29] D. Gangloff, A. Bylinskii, I. Counts, W. Jhe, and V. Vuletić, Velocity tuning of friction with two trapped atoms, Nat. Phys. 11, 915 (2015).

[30] I. Counts, D. Gangloff, A. Bylinskii, J. Hur, R. Islam, and V. Vuletić, Multislip Friction with a Single Ion, Phys. Rev. Lett. 119, 043601 (2017).

[31] M. Cetina, A. Bylinskii, L. Karpa, D. Gangloff, K. M. Beck, Y. Ge, M. Scholz, A. T. Grier, I. Chuang, and V. Vuletić, Onedimensional array of ion chains coupled to an optical cavity, New J. Phys. 15, 053001 (2013).
[32] M. Weiss and F.-J. Elmer, Dry friction in the FrenkelKontorova-Tomlinson model: Static properties, Phys. Rev. B 53, 7539 (1996).

[33] D. Leibfried, R. Blatt, C. Monroe, and D. Wineland, Quantum dynamics of single trapped ions, Rev. Mod. Phys. 75, 281 (2003).

[34] S. Aubry, The twist map, the extended Frenkel-Kontorova model and the devil's staircase, Physica D 7, 240 (1983).

[35] F. C. Frank and J. H. van der Merwe, One-dimensional dislocations. I. Static theory, Proc. R. Soc. A Math. Phys. Eng. Sci. 198, 205 (1949).

[36] S. R. Sharma, B. Bergersen, and B. Joos, Aubry transition in a finite modulated chain, Phys. Rev. B 29, 6335 (1984).

[37] B. Joos, B. Bergersen, R. J. Gooding, and M. Plischke, Commensurate and incommensurate ground states in a onedimensional model, Phys. Rev. B 27, 467 (1983).

[38] A. Socoliuc, R. Bennewitz, E. Gnecco, and E. Meyer, Transition from Stick-Slip to Continuous Sliding in Atomic Friction: Entering a New Regime of Ultralow Friction, Phys. Rev. Lett. 92, 134301 (2004).

[39] D. Gangloff, Nanocontacts controlled atom-by-atom in an ioncrystal friction emulator, Ph.D. thesis, Massachusetts Institute of Technology, 2016.

[40] A. Bylinskii, Friction under microscope with trapped ions in optical lattices, Ph.D. thesis, MIT, 2016.

[41] T. Zanca, F. Pellegrini, G. E. Santoro, and E. Tosatti, Frictional lubricity enhanced by quantum mechanics, Proc. Natl. Acad. Sci. USA 115, 3547 (2018).

[42] M. H. Müser, Velocity dependence of kinetic friction in the Prandtl-Tomlinson model, Phys. Rev. B 84, 125419 (2011).

[43] Y. Dong, A. Vadakkepatt, and A. Martini, Analytical models for atomic friction, Tribol. Lett. 44, 367 (2011). 